\title{
« LA LECTURE COMME JEU » : PRATIQUES LUDIQUES DE LA LITTÉRATURE EN RÉGIME AUDIOVISUEL
}

\author{
Marine SIGUIER $^{1}$
}

« Il n'y a pas une essence intemporelle de la littérature mais, sous le nom de littérature, un devenir de formes, de fonctions, d'institutions, de raisons, de projets fort différents. »

Roland Barthes

Cet article examine la manière dont certaines cultures médiatiques instituent des mises en scène ludiques de la littérature, de la télévision à YouTube. À la télévision, le format de l'émission littéraire s'est peu à peu cristallisé comme un programme d'information, se démarquant du «monde ludique » (Jost, 1997). À l'inverse, la plateforme de partage de vidéos YouTube, mobilisant une forte culture de la « trivialité » (Jeanneret, 2008), de la participation et de la collectivité, a rapidement constitué un espace privilégié pour « jouer » avec le littéraire. S'y développent alors de nouveaux gestes ritua-lisés qui donnent lieu à des réappropriations du livre et de sa matérialité, en périphérie des enjeux textuels traditionnellement mobilisés.

1 Marine Siguier est doctorante en sciences de l'information et de la communication au CELSA, au sein du Groupe de recherches interdisciplinaires sur les processus d'information et de communication (GRIPIC).

Recherches en communication, $\mathrm{n}^{\circ} 49$ - Article publié le 21/03/2019 
Le texte littéraire et l'image médiatique, fonctionnant sur deux régimes sémiotiques différents, sont traditionnellement associés à des valeurs opposées : écrit/oral, culture/divertissement, solennité/décontraction, élitisme/démocratisation... Au sein de la culture audiovisuelle, le livre constitue alors à la fois un « objet de résistance et un objet fétiche » (Doueihi, 2008, p. 299). Dans L'invention de la littérature, Florence Dupont (1998) oppose ainsi la culture froide du texte « culture ascétique, de la mise à distance à l'œuvre, la maîtrise de soi et la retenue [...], du commentaire plus ou moins savant ou esthétique sur les œuvres », à la culture chaude de l'oralité, « culture hédoniste de la participation et de l'identification [...], de l'engagement corporel, de la détente, du plaisir partagé, de la convivialité et de l'informalité » (Lahire, 2004, p. 73, d'après Dupont, 1998). Dans cette perspective, la mise en mots (et en images) du livre serait un premier pas vers sa mise en jeu. Le régime audiovisuel, en donnant à voir et à entendre les textes écrits, pourrait alors réactiver la dimension orale, communautaire et ontologiquement ludique de la littérature. Ce glissement paradigmatique de l'écrit à l'écran contribuerait donc à battre en brèche l'imaginaire dominant de la lecture en Occident, représentée comme « un acte par excellence du fort privé, de l'intimité soustraite au public » (Chartier, 1985, p. 96).

Cependant, la mise en scène ludique du littéraire n'est pas systématique au sein des médias audiovisuels, et reste tributaire du dispositif dans lequel elle s'inscrit. Nous verrons d'abord qu'elle est rarement mobilisée à la télévision, dans la mesure où l'émission littéraire convoque un horizon d'attente qui laisse peu de place au « monde ludique » (Jost, 1997, 2012). En revanche, d'autres espaces médiatiques, à l'instar de la plateforme YouTube, permettent l'émergence de jeux littéraires spécifiques. Nous interrogerons dans un second temps la relation existant entre ce nouveau type de médiation audiovisuelle et les dynamiques de ludification qui s'y développent, autour de vidéos publiées par les internautes. 


\section{Méthodologie}

Nous proposons de saisir les conditions de déploiement d'une mise en scène ludique du littéraire sur YouTube par une approche sémio-pragmatique. Il s'agit d'évaluer la manière dont un système médiatique préfigure un cadre technique et symbolique, au sein duquel se cristallisent des pratiques spécifiques de ludification. Dans ce cas précis, nous analyserons comment une certaine « culture YouTube »- ou "YouTube-ness » (Burgess \& Green, 2009) - permet, voire encourage, une mise en jeu des pratiques de lecture qui se donnent à voir au sein de la plateforme, à travers l'exemple des Booktubeurs. Ce terme désigne une certaine catégorie de Youtubeurs, qui utilisent la plateforme pour partager leur passion pour la littérature et parler de leurs lectures. Ces lecteurs médiatiques sont souvent des femmes d'une vingtaine d'années, qui s'adressent à un public lui aussi majoritairement jeune et féminin (Kraemer, 2016).

\begin{tabular}{|l|c|c|c|c|c|}
\hline \multicolumn{1}{|c|}{$\begin{array}{c}\text { Nom de la } \\
\text { chaîne }\end{array}$} & $\begin{array}{c}\text { Année de } \\
\text { création }\end{array}$ & $\begin{array}{c}\text { Nombre } \\
\text { de vidéos }\end{array}$ & $\begin{array}{c}\text { Nombre } \\
\text { de vues } \\
\text { cumulées }\end{array}$ & $\begin{array}{c}\text { Nombre } \\
\text { d'abonnés }\end{array}$ & $\begin{array}{c}\text { Nombre } \\
\text { de vidéos } \\
\text { «Challenge » }\end{array}$ \\
\hline $\begin{array}{l}\text { Margaud } \\
\text { Liseuse }\end{array}$ & 2007 & 437 & 4957863 & 57450 & 16 \\
\hline Nine Gorman & 2013 & 210 & 3911152 & 63703 & 16 \\
\hline Bulledop & 2012 & 164 & 2895667 & 57164 & 9 \\
\hline
\end{tabular}

Figure 1. Récapitulatif des données descriptives des chaînes analysées ${ }^{2}$

2 Données de fin décembre 2017. Nous nous sommes inspirés de la tabulation de Boris Urbas, dans « Mémoires d'une cul-ture vidéoludique sur la plateforme YouTube. Expériences de vidéastes amateurs et patrimonialisation du jeu vidéo », Les Cahiers $d u$ numérique 12(3), 2016. 
Nous chercherons à saisir ces enjeux à travers une analyse sémiologique des vidéos publiées sur les " chaînes $»^{3}$ de trois Booktubeuses françaises (Margaud Liseuse, Nine Gorman, Bulledop), de 2014 à 2017. Ces trois chaînes ont été retenues selon des critères de popularité (les trois Booktubeuses sélectionnées comptent parmi les plus suivies en France) et de pertinence du contenu (présence significative de vidéos mettant en scène des « jeux » littéraires).

Parmi les 811 vidéos initiales (total des vidéos des trois chaînes cumulées), il s'agissait ensuite de sélectionner celles qui donnaient à voir des pratiques ludiques de lecture. Pour effectuer cette sélection, nous nous sommes appuyés sur les travaux de François Jost, qui propose d'étendre les classifications traditionnelles du jeu (notamment celles de Roger Caillois) aux spécificités du média télévision. Pour ce spécialiste du petit écran, les jeux télévisuels appartiennent au monde ludique, défini comme un univers « où les émissions parlent $[\ldots]$ du monde réel, tout en se conformant à des règles propres, dont le respect est primordial (comme la fiction) » (Jost, 1999, p. 36).

Ce respect de règles spécifiques, permettant de créer un monde parallèle à mi-chemin entre le monde réel et le monde fictif, constitue le critère de base de définition du ludique chez Jost, et fait écho aux perspectives adoptées dans de nombreux autres domaines, des études littéraires (Eco, Compagnon, Dumortier, Lavandier) aux études du jeu (Juul, Jorgensen). Plusieurs chercheurs se sont ainsi penchés sur la tension ludique entre réel et imaginaire au cœur de l'activité de lecture des textes de fiction. Dans Lector in fabula, Umberto Eco montre que la lecture repose sur la relation instaurée entre le narrateur et le lecteur, où ce dernier accepte de « jouer le jeu » pour s'immerger dans le monde proposé et interagir avec le texte. Pour décrire ce phénomène, les études narratologiques empruntent au poète Coleridge le concept de «willing supsension of disbelief », ou " suspension consentie de l'incrédulité » (Compagnon, 1999; Dumortier, 2005, Lavandier, 1994). André

3 Le terme « chaîne » désigne l'espace qui regroupe toutes les vidéos publiées sous un même profil. 
Gardies étend cette conception au cinéma, à travers la notion de « spectature ludique », cette « sorte de compétition » qui s'instaure entre énonciateur et énonciataire, fondée sur « une mobilisation de l'imaginaire » lors du visionnage d'un film (Gardies, 1988). Au cinéma comme en littérature, celui qui refuse de souscrire à l'illusion proposée ne respecte pas les règles, et se met alors hors jeu.

On retrouve également cette importance du respect de règles autonomes, à mi-chemin entre monde fictif et monde réel, dans le domaine des études du jeu. Traditionnellement, et sous l'impulsion de chercheurs comme Jesper Juul (Half-real, 2005), de nombreux travaux tendent à envisager les jeux avant tout comme des systèmes au sein desquels les règles ont une place cruciale (Triclot, 2013), qui peuvent être mis en lien avec le monde fictif, mais sans y être totalement réduits (Jorgensen, 2013) ${ }^{4}$.

Ces principes fondamentaux recoupent en partie la conception de François Jost ; cependant, ce dernier s'est également attaché à saisir les spécificités du média télévision dans son rapport au ludique. Pour le chercheur, le monde ludique présente une particularité en régime télévisuel, qui s'appuie notamment sur la présence d'un double public (public participant et public regardant). En effet, le jeu télévisuel implique non seulement les candidats à l'écran, mais également les téléspectateurs «calés dans [leurs] fauteuils » (Jost, 1999), invités à jouer par procuration. De fait, à la télévision, le jeu figuré à l'écran se double d'une incitation, explicite ou non, du spectateur à reproduire lui aussi cette dynamique de jeu.

En soumettant le contenu des vidéos présélectionnées sur YouTube à ces mêmes prérequis de règlementation (règles du jeu énoncées clairement au début de chaque vidéo) et de double participation (implication directe des Booktubeurs + implication indirecte de leur public via le caractère partageable des jeux proposés), nous avons pu réduire notre corpus à un total de 41 vidéos. Cons-

4 Définissant le « gameworld», Kristin Jorgensen affirme ainsi que ce dernier peut être mis en lien avec le monde fictif, mais ne peut y être réduit, sous peine d'en écraser la complexité. Cette définition nuancée fait écho à l'ambiguïté du «monde ludique » de François Jost, oscillant en permanence entre authenticité du réel et «comme si » imaginaire. 
tatons d'emblée que ces pratiques ludiques de la littérature sont presque systématiquement regroupées sous une forme circulante sur YouTube : le « challenge », sur lequel nous reviendrons plus loin.

Par ailleurs, nous mobiliserons dans cet article le concept de médiagénie défini par Philippe Marion : est considérée comme médiagénique la capacité d'un projet narratif ou d'un genre « à se réaliser de manière optimale en choisissant le partenaire médiatique qui leur convient le mieux ». Le terme « médiagénie » est à distinguer de la " médiativité », qui désigne symétriquement ce que permet un média, soit « la capacité propre de représenter [...] qu'un média possède quasi ontologiquement » (Marion, 1997). Nous postulons que les mises en scène ludiques de la littérature sont largement tributaires du dispositif médiatique au sein duquel elles s'inscrivent. Ainsi, la télévision constitue un environnement où le jeu littéraire reste peu répandu en raison de sa faible télégénie.

\section{Le livre à la télévision : fini de jouer ?}

Dans son rapport au ministère de la Culture, Olivier Bourgois constate que la télévision est souvent associée à une menace pour les « vestales chargées d'entretenir le feu sacré du livre dans un monde étranger, voire hostile » (Bourgois, 2000, p. 5). De fait, les émissions littéraires ont cherché au fil du temps à asseoir leur légitimité en se structurant comme une émission d'information, loin de cet entre-deux entre fantasme et réel qui caractérise le jeu (Picard, 1986).

Depuis leur émergence dans les années 1950, les émissions littéraires ont connu une véritable diversité formelle, mais elles restent néanmoins tributaires d'une certaine fermeture référentielle qui permet à cette catégorie de faire sens. Le succès d'Apostrophes a notamment contribué à fixer certains invariants du dispositif (Tudoret, 2009), qui constituent encore aujourd'hui l'horizon d'attente médiatique de ce genre de programme. L'émission littéraire se décline ainsi autour d'un même modèle indépassable : une « émission d'information », appuyée sur « un dispositif de conver- 
sation » (Nel, 1996) entre auteurs. Ce modèle de cénacle médiatisé s'attache à entretenir l'illusion du réel, en effaçant certaines marques énonciatives télévisuelles pour donner l'illusion d'une médiation neutre (importance du direct, convocation de l'imaginaire du salon littéraire transposé sur un plateau, disposition des intervenants en cercle, etc.).

Ce positionnement s'oppose au fonctionnement du monde ludique, défini par François Jost comme « un "pour de rire", dans lequel la médiation se prend pour objet, qu'il s'agisse de jouer avec le langage (énonciation), de jouer avec le jeu (aléa) ou de faire de l'art pour l'art » (Jost, 2012). Au sein de l'émission littéraire, ce discours auto-référentiel est mis à distance, comme s'il revenait davantage au dispositif télévisuel de s'adapter à la littérarité des contenus, qu'à la littérature de se faire « télégénique ».

L'idée de jeu littéraire n'a pourtant pas toujours été absente du paysage télévisuel français. Dans les années 1960 à 1970, certains programmes proposaient de mettre en compétition des anonymes férus de littérature policière pour résoudre des énigmes $(A$ Malin malin et demi, 1966), des téléspectateurs pour reconnaître des œuvres à l'aide de scènes jouées par des comédiens (Quoi de qui ?, 1975), des jeunes lecteurs pour mener une enquête (Opération à livre ouvert, 1969), etc. Ces rares tentatives de mise en scène du littéraire comme jeu télévisuel ont un point commun : la figure du lecteur dit « ordinaire ». Dans toutes les émissions citées ci-dessus, les participants sont présentés comme des anonymes passionnés de lecture. En effet, contrairement au simple divertissement, le jeu télévisuel repose sur la participation (Jost, 2005, p. 93), brouillant ainsi la frontière entre « des regardants et des regardés, des spectateurs et des acteurs » (Debray, 2014). Or, la participation par délégation des spectateurs, nécessaire à la dynamique du jeu, est facilitée par « l'identification du téléspectateur à des candidats anonymes » (Jost, 2005, p. 93). Dans le cadre de l'émission littéraire, ces anonymes sont nécessairement des lecteurs, qui constituent le public modèle de ce genre de programme.

Cependant, ces jeux télévisuels font figure d'exception. Et pour cause, la majorité des émissions littéraires se construisent autour de la figure de l'écrivain, auquel il est demandé de dire et 
non de faire. Les premiers programmes culturels, dans les années 1950 et 1960, mettaient en scène des visites au " grantécrivain » (Noguez, 1995), qui recevait chez lui, " selon ses propres règles » (Tudoret, 2009, p. 46). Et si désormais « la télévision exige de (l'écrivain) qu'il se rende dans ses propres lieux, qu'il se soumette à ses propres lois, à son propre espace ", cette inversion récente (et relative) du rapport de force ne manque pas d'être assimilée à un « sacrifice sur l'autel du divertissement totalitaire » (Tudoret, 2009, p. 46). Traditionnellement, l'émission littéraire mythifie la figure du scripteur, dans la mesure où elle ambitionne de décrypter les mécanismes de la création (auteur) plutôt que les processus de la participation (lecteur), ce qui neutralise l'idée même de jeu.

Cette approche dominante de la médiation littéraire à la télévision s'inverse cependant au sein d'autres espaces médiatiques. Sur YouTube, la figure du lecteur anonyme qui « joue » à la littérature est au contraire omniprésente, et se décline selon des modalités spécifiques au dispositif.

\section{Médiagénie du jeu littéraire sur YouTube}

La plateforme de partage de vidéos organise une réalité particulière, qui fait la part belle à la notion de jeu, comme en témoigne le succès des chaînes consacrées aux jeux vidéo. En 2017, ces dernières représentent $28 \%$ des contenus consommés sur YouTube et ont généré 9,5 milliards de vues ${ }^{5}$. Cette culture ludique se reflète dans les pratiques mises en scène par la communauté des Booktubeurs, qui utilisent YouTube pour parler de leurs lectures. De nombreuses manières de « jouer » avec le littéraire s'y cristallisent, autorisées par un triple imaginaire médiatique de « trivialité $»^{6}$ (Jeanneret, 2008), de participation et de collaboration.

5 Classement réalisé en 2017 à partir du Top 100 des influenceurs (source Vidscore). Jacques Mura, YouTube : Les thé-matiques les plus consommées, médium.com, 20 février 2017.

6 Terme à distinguer de son usage péjoratif habituel, qui désigne ici la façon dont le mode de circulation des idées et des messages transforme leur sens et crée de la valeur. Nous nous référons au concept de trivialité défini par Yves Jeanneret comme " le caractère transformateur et créatif de la transmission et de la réécriture des êtres culturels à travers différents espaces sociaux » dans Critique de la trivialité. Les mé- 


\subsection{Circulation des formes ludiques sur YouTube : la trivialité du " challenge "}

À l'instar des émissions littéraires à la télévision, les Booktubeurs constituent une niche relativement restreinte au sein de l'environnement médiatique dans lequel ils s'inscrivent : les plus populaires cumulent tout juste un peu plus de 50000 abonnés, quand les plus célèbres Youtubeurs beauté, humour ou jeu vidéo dépassent fréquemment la barre du million.

Toutefois, face à ce relatif manque de médiagénie des contenus littéraires en régime audiovisuel, les stratégies médiatiques adoptées sont diamétralement opposées. Alors que l'émission littéraire sémiotise sa différence en mobilisant une rhétorique de la « forteresse assiégée » en lutte contre les logiques télévisuelles dominantes, les Booktubeurs semblent au contraire souscrire sans réserve à la puissance d'éditorialisation du dispositif. Leurs vidéos apparaissent ainsi très influencées par les nombreux codes, normes et formes circulantes sur YouTube. Cette répétition du "même " se justifie chez les Youtubeurs par une volonté de paraître attractif, en produisant un contenu stéréotypé ${ }^{7}$ :

On essaye tous de reprendre ce qui marche bien sur les autres chaînes pour les mettre sur nos chaînes à nous, parce que justement l'utilisation de ces codes permet de ne pas perdre les spectateurs, nos communautés. On leur propose quelque chose qu'elles ont déjà vu ailleurs, dans la forme, et du coup elles sont beaucoup plus réceptives que si c'était totalement différent, totalement barré, et qu'il fallait tout réintégrer. Dès qu'il y a quelque chose de nouveau, c'est toujours difficile de l'accepter, que si c'est quelque chose qui nous fait déjà penser à quelque chose d'autre. (Entretien avec Michael Roch, créateur de la chaîne YouTube La Brigade du Livre, 6 octobre 2017)

diations de la communication, enjeu de pouvoir. Paris : Éd. Non Standard, 2014, p. 15.

7 Ce terme est ici employé sans connotation négative. Nous nous référons à la définition de Ruth Amossy, qui assimile le stéréotype à " un modèle collectif figé », qui permet de se repérer « dans le foisonnement du réel ou du texte», dans Ruth Amossy, Les idées reçues. Sémiologie du stéréotype. Paris : Nathan, 1991, p. 21. 
Certaines de ces formes circulantes se distinguent par leur caractère ludique. Le « challenge » constitue ainsi un type de jeu spécifique caractérisé par sa circulation triviale : il s'agit d'accomplir un défi et d'inciter d'autres internautes à le reproduire à leur tour. Très en vogue sur de nombreux réseaux sociaux, cette forme particulière est devenue un genre à part entière sur la plateforme, mobilisée par de très nombreux Youtubeurs, toutes catégories confondues. Les Booktubeurs ne font pas exception à la règle et s'emparent régulièrement de ces contenus standardisés, pour les réinterpréter à l'aune des pratiques littéraires.

\subsection{Culture de la participation : la mobilisation du public}

YouTube est un site d'hébergement qui fonctionne en partie sur le modèle du user-generated content ou « contenu créé par les utilisateurs » : ces derniers ont la possibilité d'y publier directement leurs propres vidéos. Une "idéologie YouTube » s'élabore ainsi à partir de cette valorisation de la productivité des internautes (Burgess \& Green, 2009, p. 6). Le dispositif produit un discours d'accompagnement qui vise à soulever un " conatus discursif » (Candel, 2016, p. 324) ou désir de prise de parole chez l'usager.

Cette incitation à la participation, inhérente à la nature de cet environnement médiatique, rejaillit dans les pratiques des Youtubeurs eux-mêmes. Les Booktubeurs mobilisent leur public en l'incitant à participer à une ritualisation codifiée de ses lectures. C'est le cas du Cold Winter Challenge, organisé tous les ans par Margaud Liseuse. La Booktubeuse propose à sa " communauté » de constituer une liste de livres à lire pendant l'hiver. L'aspect ludique de cette proposition tient à une série de règles du jeu qui encadrent la participation : les lectures doivent être effectuées entre le $1^{\text {er }}$ décembre et le 31 janvier (contrainte temporelle) et un livre au minimum doit être en adéquation avec l'un des « menus de lecture » proposés par Margaud Liseuse (voir Figures 2 et 3). 


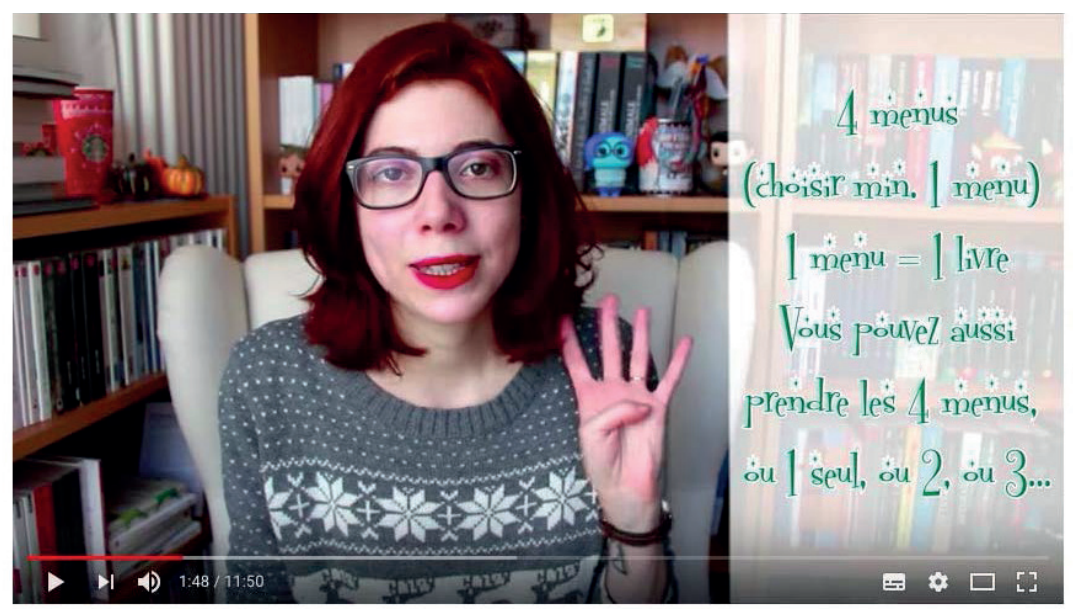

Cold Winter Challenge 2017

28380 vues

It $2 \mathrm{~K}+11 \rightarrow$ PARTAGER $\equiv+\cdots$

Margaud Liseuse

Ajoutée le 9 nov. 2017

SABONNER $57 \mathrm{~K}$

Figure 2. Capture d'écran de la vidéo Cold Winter Challenge, publiée le 9/11/2017 sur la chaîne de Margaud Liseuse

\section{Les menus}

- La magie de Noël : lire un livre en lien avec cette fête (de près ou de loin)

- Flocons magiques : lire un livre du genre fantasy, fantastique... bref magique, mais pas forcément en lien avec Noël ou l'hiver.

- Marcher dans la neige : lire un livre de natur writing, de littérature de voyage

- Stalactites ensanglantées : lire un polar/thriller qui se pase durant l'hiver, ou durant une période froide.

Figure 3. Menus thématiques proposés par Margaud Liseuse

C'est donc un imaginaire de participation et d'activité des internautes qui est ici promu, permettant la cristallisation d'une symbolique ludique. Les individus étant invités à participer par la nature même du dispositif, ils seraient ensuite à même d'y jouer 
collectivement. Aussi, « le jeu productif [...] a toujours fait partie du répertoire de ces espaces semi-privés de participation culturelle » (Burgess \& Green, 2009, p. 26), dont l'univers Booktube fait partie. De fait, il n'est pas rare d'observer chez les Booktubeurs une mise en collectivité des pratiques, qui peut se manifester par une mobilisation de leur " communauté », mais également par une collaboration entre les Booktubeurs eux-mêmes.

\subsection{Culture de la collaboration : le plaisir de la compétition amicale entre Booktubeurs}

Les chercheurs Burgess et Green constatent que les discours médiatiques présentent bien souvent les pratiques des Youtubeurs comme une nouvelle forme de téléréalité, où domine la culture du soi. Or, en réalité, la production de contenu engage aussi bien la promotion de soi que la "structuration d'un réseau social ", et donc le « jeu collectif» (Burgess \& Green, 2009, p. 29). Les stratégies de collaboration sont constitutives de l'univers YouTube (Louessard, 2017) et les Booktubeurs ne font pas exception à cette règle médiatique.

Si la lecture est un jeu, c'est d'abord un jeu solitaire, qui s'élabore entre l'individu et le texte (Picard, 1986). Dès lors, la ludification médiatique de cette activité est tributaire d'une mise en abîme, qui consiste à donner à voir ce jeu individuel à travers d'autres pratiques de jeu collectives. Cette mise en scène de la collaboration s'effectue alors à travers un double enjeu de compétition et de complicité. La mise en concurrence directe permet en effet aux Booktubeurs de confronter leurs connaissances littéraires. Elle réactive l'expérience de lecture comme "game ", terme défini par Michel Picard comme la jouissance que l'amateur éclairé ressent à reconnaître des modèles culturels (Picard, 1986). Déjà présente dans le rapport individuel aux textes, cette jouissance se trouve augmentée dans la confrontation à autrui. La dimension ludique est par ailleurs renforcée par la complicité affichée entre les Booktubeuses à l'écran, qui permet de mobiliser une 
scénographie du plaisir indissociable du jeu (« J'espère que cette vidéo vous plaira et que vous rigolerez autant que nous $\left.»^{8}\right)$.

Le triple enjeu de trivialité, de participation et de collaboration institué par la plateforme permet ainsi le déploiement de représentations de la lecture comme une activité médiatisable et communautaire, qui se " joue » à plusieurs et qui s'incarne alors dans des mises en forme particulières.

\section{Renouveler les représentations culturelles par le jeu : usages « para-littéraires » du texte}

YouTube fournit un cadre technique et symbolique au sein duquel se développent des mises en scène du littéraire spécifiques à cet environnement médiatique. Décentré du texte et de son auteur, le plaisir littéraire se déploie en périphérie de la lecture elle-même, à travers des scénographies à la fois inédites et tributaires de formes antérieures de représentations du littéraire.

\subsection{Renouveler la matérialité de l'objet livre : le Book Tower Challenge}

Certains usages subvertissent ainsi totalement la valeur symbolique du livre, en le rattachant à sa dimension matérielle d'objet. C'est le cas du Book Tower Challenge : il s'agit d'ériger, en un temps limité et selon des critères précis, une pile de livres sans que celle-ci ne s'effondre (voir Figure 4).

Le jeu permet ici de rendre le texte imprimé médiagénique autrement que par sa lecture. Les livres y sont paradoxalement rendus ludiques car ils sont ramenés à leur nature de " parallélépipèdes qui ne bougent pas, ne parlent pas ", selon la formule de Bernard Pivot (Cahiers du cinéma, automne 1981). Il ne s'agit plus de les transcender par l'image et le son, mais, au contraire, de les donner à voir dans leur fonction la plus prosaïque d'objets inertes que l'on empile. Cet usage utilitaire fait écho à la fétichi-

8 Nine Gorman, « Harry Potter Lemon Challenge », YouTube, 14 juillet 2015, URL : https://www.youtube.com/watch?v=9VRLNatienE 


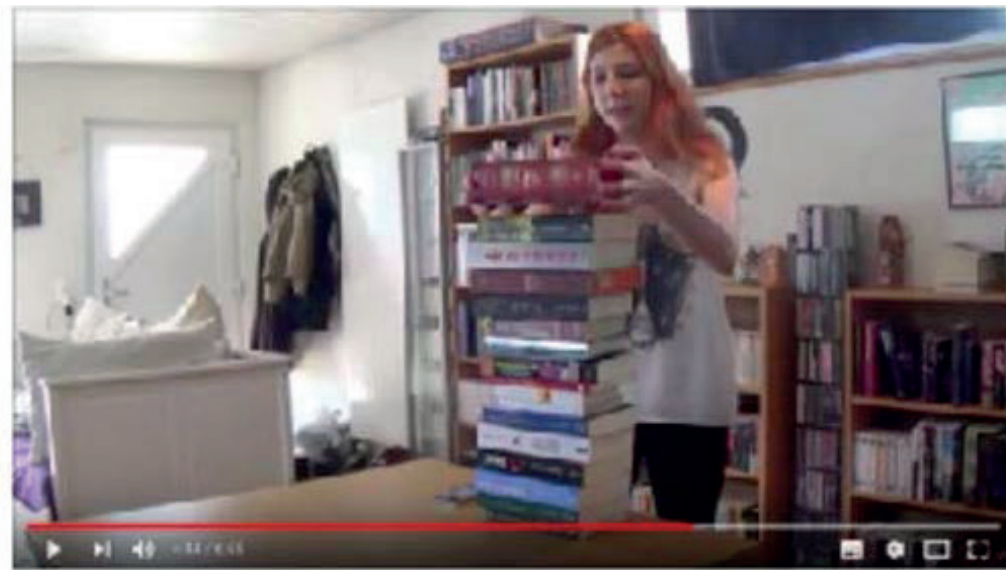

Bock Tuve Chylariget

Noni net

Figure 4. Capture d'écran de la vidéo Book Tower Challenge, publiée le 18/05/2014 sur la chaîne de Margaud Liseuse

sation de l'objet dans les émissions littéraires, qui utilisent le livre comme décor, et où la bibliothèque en arrière-plan sert avant tout à incarner la fonction-livre sur le plateau.

\subsection{Dépasser l'irréductibilité sémiotique : l'Emoticone Book Challenge}

À l'opposé, il existe également des mises en scène du texte qui cherchent à résoudre cette " irréductibilité sémiotique » (Souchier, 2015) en mobilisant un langage audiovisuel spécifique pour « faire parler » les textes. C'est le cas de l'Emoticone Book Challenge : il s'agit de faire deviner le titre d'un roman en utilisant uniquement les petits symboles iconiques d'une application de messagerie (une jeune fille, un lapin et une montre pour évoquer Alice au pays des merveilles par exemple : voir Figure 5). Cette mise en jeu s'appuie sur certains codes institués par les nouvelles formes numériques, qui exploitent la dichotomie entre textualité du livre et iconicité d'une certaine « culture numérique » (Doueihi, 2008). 


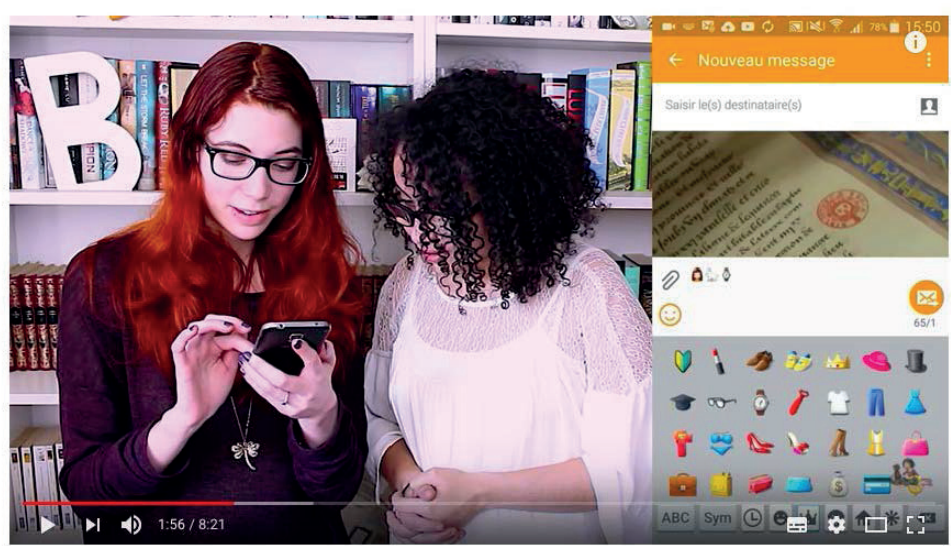

Emoticones Book Challenge | Ft. @Margaudliseuse 21417 vues

(4) Bulledop

Figure 5. Capture d'écran de la vidéo Emoticones Book Challenge, publiée le 27/05/2016 sur la chaîne de Bulledop.

Cette mise en image des textes littéraires dans une perspective ludique fait écho aux formats télévisuels évoqués plus haut. La posture de lecteur « ordinaire » qui doit identifier des textes incarnés à l'image rappelle ainsi le principe de jeux télévisuels tels que Quoi de qui ?, émission diffusée en France en 1975, où les candidats devaient reconnaître les œuvres littéraires évoquées à l'aide de scènes pastiches jouées par des comédiens. La différence principale réside ici dans le fait que l'incarnation du texte s'effectue non plus par une performance théâtrale, mais par la mobilisation de nouvelles formes iconiques, qui sont utilisées pour résumer la structure narrative du roman à travers l'usage d'un langage minimaliste.

\subsection{Mobiliser son capital culturel autrement : l'Infinite Book Challenge}

La ludification des pratiques de lecture permet également un détournement axiologique des enjeux de la « culture cultivée », à 
l'instar de 1'Infinite Book Challenge, qui consiste à énumérer le plus de titres de roman possible en une minute.

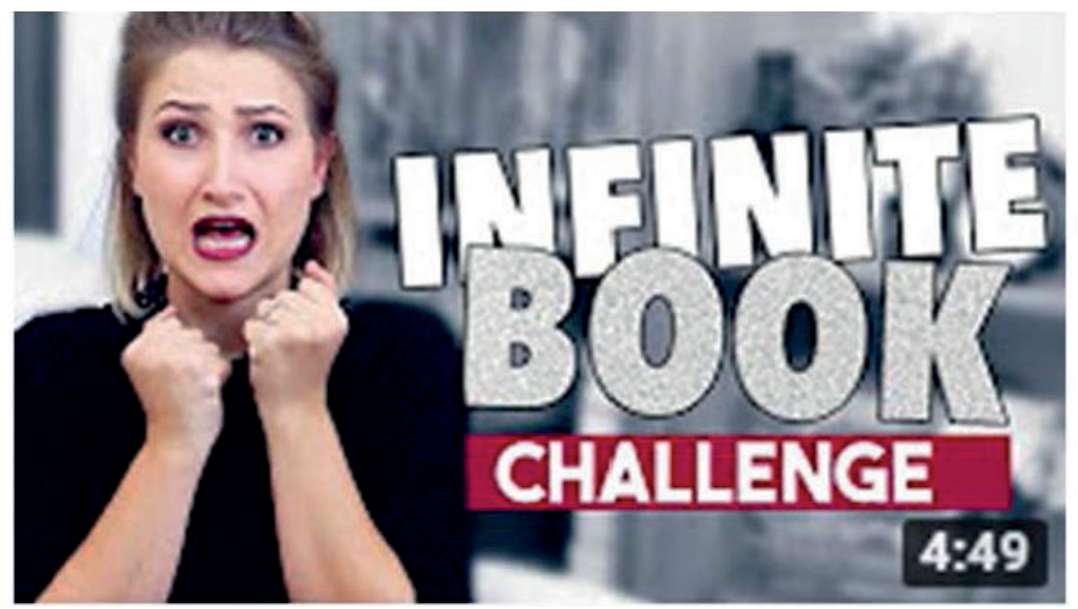

Figure 6. Capture d'écran de la vidéo Infinite Book Challenge publiée le 3 octobre 2015 sur la chaîne de Nine Gorman

Ce type de challenge valorise l'aspect quantitatif des lectures, en adéquation avec la médiagénie du chiffre sur Internet, où les algorithmes " jettent leur filet sur le monde pour le rendre mesurable en tout » (Cardon, 2015). Cette valorisation quantitative s'oppose à la traditionnelle valorisation qualitative des références littéraires dans les médias : à la télévision, l'auteur est ainsi souvent invité à évoquer en détail son livre préféré, la plupart du temps issu du répertoire des " classiques ». Le dispositif institue de fait une certaine idée de ce qui constitue un bon partage de la littérature, en adéquation avec la médiaculture qui lui est propre.

\section{Conclusion}

L'inscription des enjeux littéraires au sein d'une culture audiovisuelle engage des mises en scène spécifiques. Le régime du son et de l'image, en donnant à voir les textes autrement, autorise des scénographies articulées autour d'une certaine mise en jeu de la littérature, dont la médiagénie varie en fonction des contextes et des dispositifs. 
De fait, le mariage du livre et de l'audiovisuel ne va pas de soi, car il mobilise des symboliques traditionnellement opposées (jeu solitaire/cérémonial collectif, culture froide/culture chaude, etc). Face à cette dichotomie des imaginaires, le format de l'émission littéraire s'est peu à peu sédimenté comme un programme d'information. Appuyée sur un modèle réaliste de conversation entre écrivains, la " promesse ontologique » (Jost, 1997) de ce type de programme désamorce l'idée même de jeu, à l'exception de rares tentatives novatrices et éphémères.

Sur YouTube, en dépit de positionnements équivalents (contenus littéraires « de niche » au sein d'un paysage audiovisuel très diversifié), la situation est diamétralement opposée. En mobilisant une forte culture de la trivialité, de la participation et de la collectivité, la plateforme a rapidement été instituée comme espace privilégié pour la médiatisation de contenus ludiques. Les pratiques des Booktubeurs, loin de s'inscrire dans une logique de résistance, se développent en adéquation avec cet imaginaire du jeu, en s'appropriant des formes circulantes telles que le challenge.

Ces nouveaux gestes ritualisés donnent lieu à des réappropriations du livre spécifiques, en périphérie du texte littéraire luimême. En apparence inédits, voire parfois déroutants, ces usages réinvestissent des enjeux de matérialité, d'iconicité et de capital culturel tributaires d'une mémoire audiovisuelle de la médiation littéraire, ré-émergeant ici sous une autre forme et dans un autre espace. Pour autant, ce type de pratique extra-littéraire ne prétend pas se substituer à des activités de critique du texte en lui-même. Parallèlement à la « frivolité » assumée et revendiquée des challenges, les Booktubeurs s'attachent par ailleurs à mettre en scène un rapport plus traditionnel et plus direct au récit, livrant leur opinion sur l'écriture, l'histoire, les personnages, etc. Le challenge constitue ainsi avant tout un mode de médiatisation complémentaire et cette alliance d'analyses textuelles et de jeux plus éloignés d'une littérarité stricto sensu contribue à faire circuler - et donc vivre une « certaine idée » (Peytard, 1990) du partage littéraire au sein de l'espace social. 


\section{Références}

Bourgois, O. (2000). Le livre à la télévision. Rapport pour le ministère de la Culture et de la Communication.

Burgess, J. \& Green, J. (2009). YouTube : Online video and participatory culture. Cambridge : Polity Press.

Candel, E. (2016). Le cas de la critique de livre « participative » sur les réseaux. Dans I. Rialland (dir.). Critique et médium (pp. 321-335). Paris : CNRS Éditions.

Cardon, D. (2015). À quoi rêvent les algorithmes? Nos vies à l'heure des big data. Paris : Seuil et La République des Idées.

Chartier, R. (1985). Pratiques de la lecture. Paris : Éditions Rivages.

Compagnon, A. (1999). Brisacier, ou la suspension de l'incrédulité. Colloque Fabula Les Frontières de la fiction.

Daney, S. \& Touiana, S. (1981). Entretien avec Bernard Pivot. Cahiers du cinéma 328, numéro spécial « Télévision », 68-71.

Doueihi, M. (2008). La grande conversion numérique. Paris : Seuil.

Dumortier, J.-L. (2005). Petit traité de narratologie buissonnière. Namur : Presses universitaires de Namur.

Dupont, F. (1998). L'invention de la littérature De l'ivresse grecque au livre latin. Paris : La Découverte.

Eco, U. (1979). Lector in fabula. Le rôle du lecteur ou la coopération interprétative dans les textes narratifs. Paris : Éditions Grasset.

Gardies, A. (1988). Le pouvoir ludique de la focalisation. Protée 16(1-2), 139-144.

Jeanneret, Y. (2008). Penser la trivialité. Volume 1 : La vie triviale des êtres culturels. Paris : Éditions Hermès-Lavoisier.

Jorgensen, K. (2013). Gameworld interfaces. Cambridge : MIT Press.

Jost, F. (1997). La promesse des genres. Réseaux 15(81), 13-31.

Jost, F. (1999). Introduction à l'analyse de la télévision, Paris : Ellipses

Jost, F. (2005). Comprendre la télévision et ses programmes. Paris : Armand Colin.

Jost, F. (2012). La promesse des genres. Comment regardons-nous la télévision? Rastros Rostros 14(27), 25-35.

Juul, J. (2005). Half-real. Video games between real rules and fictional worlds. Cambridge : MIT Press.

Kraemer, V. (2016). Qui sont les abonnés des Booktubers ? Lecture Jeune 158.

Lahire, B. (2004). La culture des individus. Dissonances culturelles et distinction de soi. Paris : La Découverte.

Lavandier, Y. (1994). La dramaturgie. Cergy : Le Clown et l'Enfant.

Leveneur, L. (2009). Les travestissements des jeux télévisés, histoire et analyse d'un genre protéiforme. Paris : Éd. Presses Sorbonne Nouvelle.

Louessard, B. (2017). La figure du youtubeur'se au cœur des mondes de l'art. Le cas des youtubeur'se's de fiction francophone. Actes du colloque Youtubeurs, Youtubeuses. Figures - formats - savoirs - pouvoirs, Tours.

Marion, P. (1997). Narratologie médiatique et médiagénie des récits. Recherches en communication 7, 61-88.

Monvallier (de), H. (2014). Entretien avec Régis Debray : autour du stupéfiant image. Actu Philosophia.

Nel, N. (1996). Écrans et dispositifs de littérature à la télévision. CinémAction 79. 
Noguez, D. (1995). Le Grantécrivain. Le Débat 4(86), 39-51.

Peytard, J. (1990). La médiacritique littéraire à la télévision. Semen 5, 105-190.

Picard, M. (1986). La Lecture comme jeu. Paris : Éditions de Minuit.

Souchier, E. (2015). Le carnaval typographique de Balzac. Premiers éléments pour une théorie de l'irréductibilité sémiotique. Communication \& langages 3(185), 3-22.

Triclot, M. (2013). Game studies ou études du play ? Une lecture croisée de Jacques Henriot et de Jesper Juul. Sciences du jeu 1. Disponible à : http://journals.openedition.org/sdj/223. Doi : 10.4000/sdj.223.

Tudoret, P. (2009). L'écrivain sacrifié. Vie et mort de l'émission littéraire. Paris : Le Bord de l'eau. 


\section{(c) $\left(\begin{array}{l}\text { BY } \\ \text { NC ND }\end{array}\right.$ Publié sous la licence Creative Commons}

«Attribution - Pas d'Utilisation Commerciale - Pas de Modification 4.0 International» (CC BY-NC-ND) 